\title{
Experimental observation of a nonpitchfork acceleration instability in a nematic liquid crystal
}

\author{
Matthias Müller,* Wolfgang Schöpf, ${ }^{\dagger}$ and Ingo Rehberg ${ }^{\ddagger}$ \\ Experimentalphysik V, Universität Bayreuth, D-95440 Bayreuth, Germany
}

(Received 23 February 2007; revised manuscript received 20 July 2007; published 24 October 2007)

\begin{abstract}
The velocity of domain walls caused by a symmetry-breaking instability is experimentally investigated for the case of electroconvection in a nematic liquid crystal. It turns out that the velocity increases linearly with the distance from the bifurcation creating the domains. This scaling behavior does not agree with the drift pitchfork bifurcation. It has, however, been predicted by a minimal model describing our scenario.
\end{abstract}

DOI: 10.1103/PhysRevE.76.041704

PACS number(s): 61.30.Gd, 47.20.Ky, 47.20.Lz, 47.65.-d

\section{INTRODUCTION}

The spontaneous onset of motion accompanied by spatial symmetry breaking, the so-called acceleration instability, emerges in a great variety of systems. Such transitions can be observed, for example, in the running of droplets on a chemically activated substrate $[1,2]$, in the motion of patterns in gas discharge systems [3-5], in the motion of Bloch walls [6], or in the acceleration of spiral cores described by the complex Ginzburg-Landau equation [7]. All these instabilities are drift pitchfork bifurcations; i.e., the velocity of the system scales with the square root of the control parameter. This paper presents an experimental study of a different, nonpitchfork bifurcation behavior.

The ac-driven electroconvection in planarly aligned nematic liquid crystals is a prime example of pattern-forming instabilities. In this system the threshold and near-threshold behavior has been largely clarified (for reviews see [8-12]). This paper deals with moving domain walls [as shown in Fig. 1(c)] that may arise as a secondary instability, depending on material parameters and on the frequency and amplitude of the applied field.

In the liquid-crystal system considered here, the fluid is sandwiched between two parallel and electrically conducting boundaries which, through special treatment, impose a preferred direction on the nematic director. Below the critical voltage $U_{n r}$ for the primary threshold, the director is aligned parallel everywhere in the cell-namely, in that direction which is imposed by the planar alignment [here the $x$ direction; see Fig. 1(a)]. The normal roll instability that occurs for $U>U_{n r}$ is the result of a periodic deformation of the director field in the $x-z$ plane [see Fig. 1(b)]. The roll axis is perpendicular to the preferred direction while the projection of the director onto the horizontal boundaries is still in the preferred direction. When increasing the driving voltage, it was found that normal rolls may be destabilized through the wellknown zigzag instability [13-16], but only for frequencies $f$ below a certain value $f_{a r}$. For higher frequencies, a homogeneous symmetry-breaking instability occurs at a critical voltage $U_{a r}[17,18]$ in the form of the so-called abnormal rolls, which have an additional component of the director along the

\footnotetext{
*matthias.mueller1@uni-bayreuth.de

†wolfgang.schoepf@uni-bayreuth.de

*ingo.rehberg@uni-bayreuth.de
}

$y$ direction. The projection of the director onto the horizontal boundaries now has an angle with the preferred direction while the roll axis is still perpendicular to the preferred direction. Due to the planar anchoring, the rotation vanishes at the horizontal boundaries, leading to a twist deformation of the director field. For symmetry reasons, this deformation can be either right or left handed, leading to the coexistence of domains for the two possibilities, which are separated by domain walls [see Fig. 1(c)]. The zigzag instability and the abnormal roll instability meet in a codimension- 2 point where further lines emerge, leading to a rather complex and interesting bifurcation scenario (see Fig. 2). This has been studied experimentally and theoretically in some detail $[19,20]$.
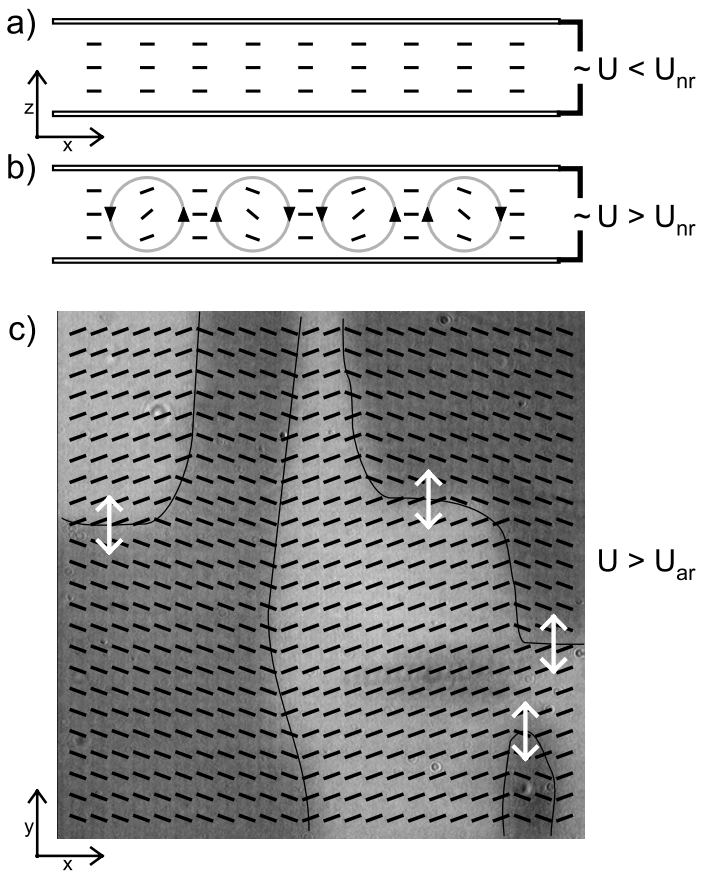

FIG. 1. Manifestation of the various patterns and definition of the cell geometry and coordinate system. (a) Cross section of the cell in the ground state. The black bars indicate the director orientation. (b) Cross section of the cell above threshold, illustrating the director configuration in convection rolls. (c) Top view of the cell obtained by ellipsometrical optics in the abnormal roll state. The black bars indicate the abnormal roll director orientation, the lines the domain walls, and the white arrows the motion of the domain walls. 


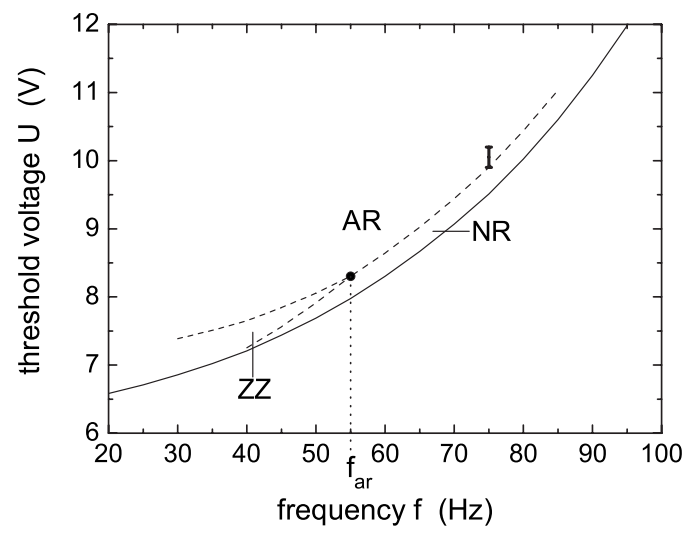

FIG. 2. Schematic phase diagram of the bifurcation scenario. The solid line marks the primary instability where the normal roll (NR) pattern emerges. The transitions to the zigzag (ZZ) pattern and to the abnormal rolls (AR) are indicated by the dashed lines. The solid dot represents the codimension-2 point at the critical frequency $f_{a r}$, and the bar at $75 \mathrm{~Hz}$ shows the range of our measurements.

Abnormal rolls can be generated from normal rolls in one of two ways. For frequencies below $f_{a r}$, zigzag rolls are the precursor to abnormal rolls. When the voltage is increased, the zigzag angle decreases continuously to zero, and at some point, abnormal rolls stabilize. For frequencies above $f_{a r}$, there is a direct transition from normal to abnormal rolls. In this paper, we study the dynamic properties of the abnormal roll domain walls in the latter case-namely, the correlation between their velocity and the driving voltage, which is expected to show an interesting scaling behavior according to theoretical predictions [20].

\section{EXPERIMENTAL SETUP}

We use the standard liquid crystal N-(4-methoxybenzylidene)-4-butylaniline (MBBA), which has a positive anisotropy of the conductivity and therefore shows electroconvection. The typical experimental setup is used, as described, e.g., in Ref. [12], and the MBBA is prepared in commercially available cells (E.H.C. Co. Ltd., 1164 Hino, Hino-shi, Tokyo, Japan 191). They consist of two parallel glass plates, which are separated by spacers and which have an indium tin oxide (ITO) layer on the inside. The ITO surfaces of the electrodes are coated with a polymer and rubbed in one direction in order to produce the planar alignment of the liquid crystal: when the cell is filled with a nematic material, the director is aligned along the rub direction in the layer plane. This direction defines the $x$ axis, with the $y$ axis being perpendicular to $x$ in the layer plane. The thickness of the cell $d=(25 \pm 1) \mu \mathrm{m}$ in the $z$ direction is determined by the spacers between the glass plates.

A sinusoidal ac voltage $U_{\text {appl }}(t)=U \sqrt{2} \cos (2 \pi f t)$ at a frequency of $f=75 \mathrm{~Hz}$ is applied across the electrodes by means of a wave-form generator. The cell is illuminated by a lightemitting diode (SHARP GL0ZS042B0S) with a wavelength of $\lambda=609 \mathrm{~nm}$ and a spectral bandwidth of $\Delta \lambda=15 \mathrm{~nm}$. The patterns are observed with a polarizing transmission micro- a)

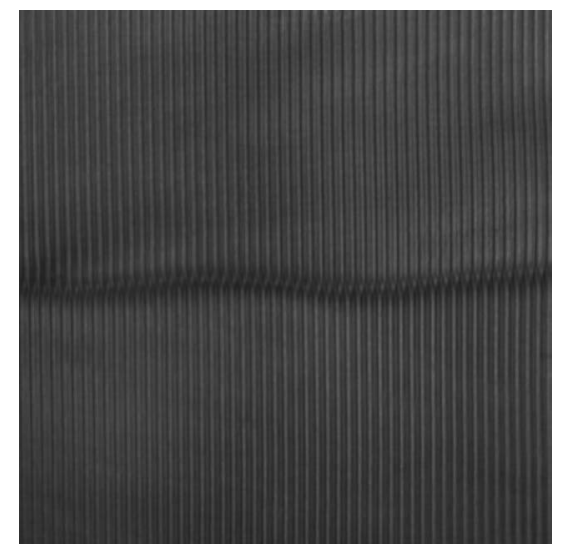

b)

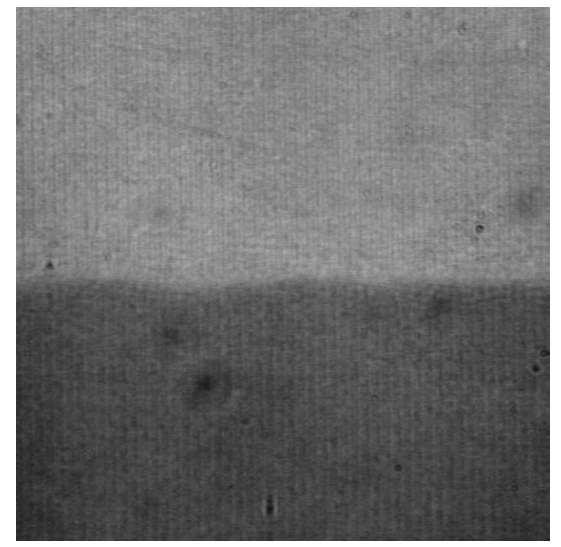

FIG. 3. Visualization of two domains of abnormal rolls with opposite twist. (a) If the incoming light is linearly polarized along the director, the rolls are clearly visible. (b) If an ellipsometrical method as described in the text is used, the different twist deformations can be distinguished by the different intensities. The domain wall is obvious in both cases.

scope and recorded with a charge-coupled-device (CCD) camera that is connected to a frame grabber card. The images have a physical size of $1.4 \times 1.4 \mathrm{~mm}^{2}$ and are digitized with a spatial resolution of $512 \times 512$ pixels into 256 grayscale values. The measurements are performed at a temperature of $T=20{ }^{\circ} \mathrm{C}$ which is stabilized to $\pm 5 \mathrm{mK}$.

If the convection cell is illuminated with light linearly polarized along the director-i.e., in the $x$ direction-the convection rolls (normal or abnormal) are visible as shown in Fig. 3(a). Two domains of abnormal rolls with opposite twist deformation, separated by a domain wall, can be seen. The twist deformation of the director caused by the abnormal rolls is not visible here. In order to visualize this twist, we use an ellipsometrical optical method as described in Refs. [21-23]. Now the convection cell is illuminated with light linearly polarized perpendicular to the director-i.e., in the $y$ direction. Since the planar alignment forces the director to be parallel to the $x$ axis on both glass plates and since the polarization plane of the light adiabatically follows the director twist [24], the direction of polarization should still be in the $y$ direction after the light has passed through the sample. As shown in Ref. [23], however, the circularity of the light changes, depending on the maximum twist angle. Using a 


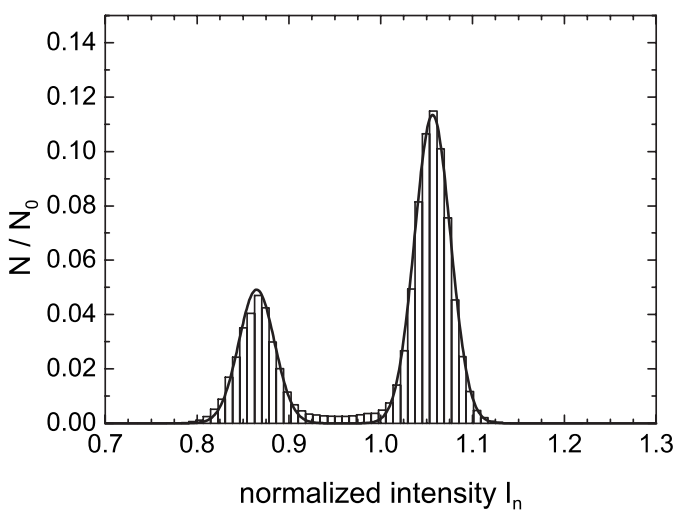

FIG. 4. Histogram for the intensities of a series of 4400 images taken at $U=10.23 \mathrm{~V}$. The solid line shows the result of the fit of Eq. (1). $N$ refers to the number of counts and $N_{0}$ to the number of pixels within an image.

circular analyzer - the inverse of a circular polarizer; see Ref. [23] —at an angle of $45^{\circ}$ with the $x$ axis, the difference in circularity manifests itself in a change of the intensity of the transmitted light. In this way we can distinguish between the left- and right-handed twists as shown by the different intensities of the two domains in Fig. 3(b). With this setup, however, the rolls themselves are hardly visible.

\section{DATA ANALYSIS AND RESULTS}

All measurements are done with the ellipsometrical optics at a frequency of $75 \mathrm{~Hz}$. The frequency $f_{a r}$ of the codimension-2 point is found between $50 \mathrm{~Hz}$ and $55 \mathrm{~Hz}$, so that we are in the regime of a direct transition from normal to abnormal rolls. The voltage is varied in the vicinity of the abnormal roll onset. For each voltage, we take a series of 4400 full images with one image taken every $2.5 \mathrm{~s}$. Two full sets of such measurements have been performed in the same cell, with 1 month elapsed between the two sets. After the second set of measurements was finished, the threshold for the onset of normal rolls has been determined by the normal shadowgraph method [25] as $U_{n r}=9.51 \mathrm{~V}$.

\section{A. Onset of abnormal rolls}

In order to determine the onset of abnormal roll convection, we measure the difference in intensity between the light and dark domains [similar to those in Fig. 3(b)] as a function of the voltage. The data are normalized by dividing each image pixelwise by a background image $I_{0}(x, y)$, which has been obtained at a voltage of $U=0.1 \mathrm{~V}$. The histogram of the normalized intensity $I_{n}(x, y)=I(x, y) / I_{0}(x, y)$ generated from the 4400 images of a series is presented in Fig. 4. We search for the two maxima in these histograms, which correspond to the different domains. This is done by fitting a superposition of two Gaussian curves to the histogram (see Fig. 4):

$$
\begin{aligned}
f\left(I_{n}\right)= & \frac{W}{\sigma \sqrt{2 \pi}}\left\{a \exp \left[-\frac{1}{2}\left(\frac{I_{n}-I_{r}}{\sigma}\right)^{2}\right]\right. \\
& \left.+(1-a) \exp \left[-\frac{1}{2}\left(\frac{I_{n}-I_{l}}{\sigma}\right)^{2}\right]\right\},
\end{aligned}
$$

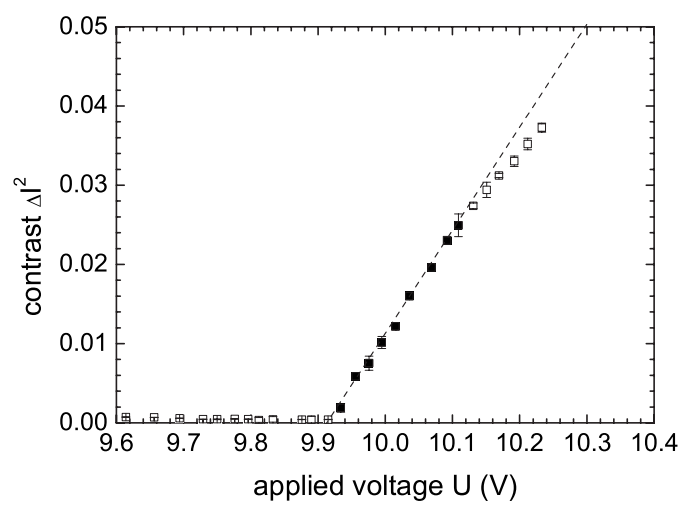

FIG. 5. Squared contrast of abnormal roll domains as a function of the applied voltage. The dashed line is the result of a linear regression performed with the data points represented by the solid symbols.

where $W$ is a normalization factor which represents the width of one histogram bar. $I_{r}, I_{l}, \sigma$, and $a$ are the fit parameters. A measure for the optical contrast is given by $\Delta I=I_{r}-I_{l}$.

The result of this contrast analysis is presented in Fig. 5, where the squared intensity difference $\Delta I^{2}$ is shown for the first of our two sets of measurements. For the two measurement series, we find $U_{a r}=9.91 \mathrm{~V}$ and $9.89 \mathrm{~V}$, respectively; i.e. the threshold has shifted by about $0.2 \%$ within 1 month. The control parameter $\varepsilon$ for abnormal rolls is then defined as

$$
\varepsilon=\frac{U-U_{a r}}{U_{a r}} .
$$

Due to an uncertainty of about $\pm 0.002 \mathrm{~V}$ for the voltage measurements, the error for $\varepsilon$ is approximately \pm 0.0004 .

\section{B. Motion of the domain walls}

We are interested in the motion of horizontal domain walls-i.e., walls parallel to the $x$ direction. The analysis of the picture series is performed in three steps. First, we have to find the domain walls in the individual images. For this purpose, each vertical line in an image is scanned for intensity changes of the transmitted light and neighboring changes are collected into walls, thereby making sure that only horizontal wall pieces are selected. Second, we examine if walls are found at similar positions in consecutive pictures. If they are identified to be one and the same wall, the velocity of this wall is determined in the final step. From the absolute values of the velocities obtained in this way from all 4400 images of a series we calculate the mean domain wall velocity.

These velocities are shown in Fig. 6 for both sets of measurements, represented by two different symbols. Each data point was measured 4 times, and the results were averaged. The error bars represent the corresponding standard deviation of these four measurements. For $\varepsilon<0.015$, the data points lie on a straight line passing through the origin, while for larger values of $\varepsilon$, the velocities are below this line. It is important to note that the fit producing this line was not forced to pass through the origin, which is a confirmation of the threshold voltage $U_{a r}$ obtained from the contrast measurements discussed earlier. 


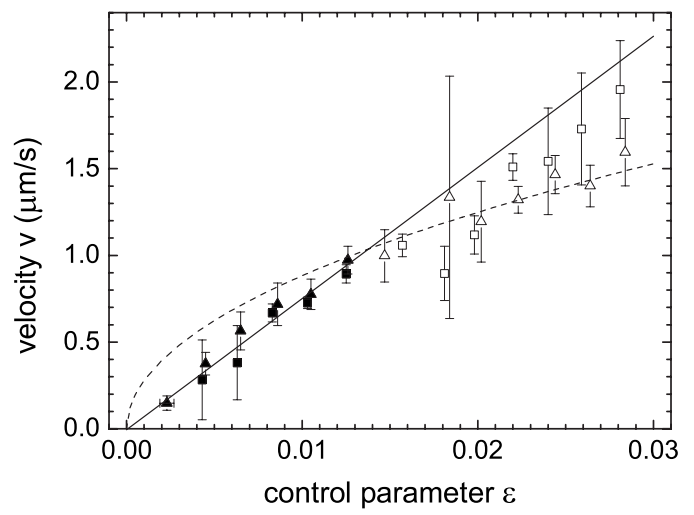

FIG. 6. Mean velocity of horizontal domain walls as a function of the control parameter $\varepsilon$. The squares and triangles represent our two sets of measurements, which have been taken with 1 month in between. The error for $\varepsilon$ is the same for all the data and is thus shown only for the leftmost data point. The solid line is the result of a linear regression performed with the data points represented by the solid symbols. For comparison, a square-root fit to the data points is also shown (dashed line).

\section{DISCUSSION AND CONCLUSION}

For an acceleration instability as investigated here, a pitchfork bifurcation might be expected, where the velocity is proportional to the square root of the distance from the bifurcation point [5]. To cross-check this behavior, we also fitted a square-root function to our data. Forcing the fit to pass through the origin, as shown by the dashed line in Fig. 6 , there is a systematic deviation from the measured data, especially close to the bifurcation point. If, on the other hand, the origin of the square root fit is not fixed, the intersection with the $\varepsilon$ axis occurs at $\varepsilon \approx 0.004$. This does not agree with our experiment showing motion above $\varepsilon=0$. In summary, we can conclude that in the vicinity of the onset, the bifurcation scenario of the domain wall instability is better described by a linear dependence on the control parameter than by a pitchfork model.

Such an unusual behavior has been numerically obtained for the case of the transition from normal to abnormal rolls via the zigzag roll regime-i.e., for frequencies $f<f_{\text {ar }}$ [20]. Although this theoretical analysis has been done for parameters slightly different from those of our experiments, it seems that a qualitative explanation is provided by the model presented in Ref. [20]. Thus the measurements presented here are in agreement with the theoretical prediction for this new type of acceleration instability.

In principle, the velocity of the domain walls is also expected to be influenced by their slope and curvature. Taking into account the long time it took to gather the data even for the simplest case of straight walls along the director, we believe that a new technique will be needed for a systematic investigation of this question. A direct "printing" technique for the walls, possibly by making use of external magnetic fields, could be helpful here.
[1] U. Thiele, K. John, and M. Bär, Phys. Rev. Lett. 93, 027802 (2004).

[2] K. Krischer and A. Mikhailov, Phys. Rev. Lett. 73, 3165 (1994).

[3] E. Ammelt, D. Schweng, and H.-G. Purwins, Phys. Lett. A 197, 348 (1993).

[4] P. Schütz, M. Bode, and V. V. Gafiichuk, Phys. Rev. E 52, 4465 (1995).

[5] M. Or-Guil, M. Bode, C. P. Schenk, and H.-G. Purwins, Phys. Rev. E 57, 6432 (1998).

[6] P. Coullet J. Lega, B. Houchmanzadeh, and J. Lajzerowicz, Phys. Rev. Lett. 65, 1352 (1990).

[7] I. Aranson, L. Kramer, and A. Weber, Phys. Rev. Lett. 72, 2316 (1994).

[8] M. C. Cross and P. C. Hohenberg, Rev. Mod. Phys. 65, 851 (1993).

[9] S. Kai and W. Zimmermann, Prog. Theor. Phys. Suppl. 99, 458 (1989).

[10] L. Kramer and W. Pesch, Annu. Rev. Fluid Mech. 27, 515 (1995).

[11] A. Buka and L. Kramer, Pattern Formation in Liquid Crystals (Springer, New York, 1996).
[12] I. Rehberg, B. L. Winkler, M. de la Torre Juárez, S. Rasenat, and W. Schöpf, Adv. Solid State Phys. 29, 35 (1989).

[13] E. Braun, S. Rasenat, and V. Steinberg, Europhys. Lett. 15, 597 (1991).

[14] S. Nasuno and S. Kai, Europhys. Lett. 14, 779 (1991).

[15] S. Nasuno, O. Sasaki, S. Kai, and W. Zimmermann, Phys. Rev. A 46, 4954 (1992).

[16] M. Kaiser and W. Pesch, Phys. Rev. E 48, 4510 (1993).

[17] E. Plaut, W. Decker, A. G. Rossberg, L. Kramer, W. Pesch, A. Belaidi, and R. Ribotta, Phys. Rev. Lett. 79, 2367 (1997).

[18] E. Plaut and W. Pesch, Phys. Rev. E 59, 1747 (1999).

[19] S. Rudroff, H. Zhao, L. Kramer, and I. Rehberg, Phys. Rev. Lett. 81, 4144 (1998).

[20] H. Zhao and L. Kramer, Phys. Rev. E 62, 5092 (2000).

[21] M. Grigutsch, N. Klöpper, H. Schmiedel, and R. Stannarius, Mol. Cryst. Liq. Cryst. Sci. Technol., Sect. A 261, 283 (1995).

[22] H. Amm, R. Stannarius, and A. G. Rossberg, Physica D 126, 171 (1997).

[23] S. Rudroff, V. Frette, and I. Rehberg, Phys. Rev. E 59, 1814 (1999).

[24] C. Mauguin, Bull. Soc. Fr. Mineral. 34, 71 (1911).

[25] S. Rasenat, G. Hartung, B. L. Winkler, and I. Rehberg, Exp. Fluids 7, 412 (1989). 